\author{
Ecología
}

\title{
Composición y actividad de la comunidad de murciélagos artropodívoros en parques eólicos del trópico mexicano
}

\author{
Composition and activity of arthropodivorous bat community in wind farms in the Mexican Tropics \\ Margarita García-Luis* y Miguel Briones-Salas
}

Laboratorio de Vertebrados Terrestres (Mastozoología), Centro Interdisciplinario de Investigación para el Desarrollo Integral Regional, Unidad Oaxaca (CIIDIR-Oaxaca), Instituto Politécnico Nacional, Hornos 1003, 71230 Santa Cruz Xoxocotlán, Oaxaca, México

Recibido el 6 de julio de 2016; aceptado el 3 de mayo de 2017

Disponible en Internet el 21 de noviembre de 2017

\begin{abstract}
Resumen
Se estudió la composición y la actividad de murciélagos artropodívoros, durante las temporadas de lluvias y secas de 2013 y 2014 , para evaluar el efecto de los parques eólicos sobre estas especies. Se capturaron ejemplares con redes de niebla y se grabaron sonidos de ecolocación en vuelo libre con un detector ultrasónico en 2 áreas: dentro de parques eólicos (DPE) y fuera de parques eólicos (FPE). Se registraron 13 especies y 4 géneros. En las redes se registraron 100 individuos de 8 especies (con un esfuerzo de muestreo de 120,600 m.red/h); con el detector, se grabaron 4,518 pases de 9 especies y 4 géneros. En el área DPE se registraron 12 especies y 4 géneros; en el área FPE, 10 especies y 4 géneros. El análisis no mostró diferencias significativas para la actividad entre las áreas DPE y FPE; no obstante, por temporadas sí se encontraron diferencias. Durante las 19:00 y 20:00 h, en ambas condiciones se registraron el mayor número de pases. Es posible que las variables ambientales y del paisaje que han sido reportadas de importancia para la actividad de los murciélagos intervengan en esta región al no encontrarse diferencias en el análisis realizado. (C) 2017 Universidad Nacional Autónoma de México, Instituto de Biología. Este es un artículo Open Access bajo la licencia CC BY-NC-ND (http://creativecommons.org/licenses/by-nc-nd/4.0/).
\end{abstract}

Palabras clave: Anabat; Detección ultrasónica; Eventos de caza; Horarios de actividad; Istmo de Tehuantepec

\section{Abstract}

We studied the bat arthropodivorous species composition and their activity during the rainy and dry seasons of 2013 and 2014 , to evaluate the effect of wind farms on these species. We captured specimens using mist nets and we recorded echolocation sounds a free fly with an ultrasonic detector in 2 areas: with wind farms (DPE) and without it (FPE). We recorded 13 species and 4 genera. In mist nets we registered 100 individuals from 8 species (with a sampling effort of 120,600 m.mist net/hr); using an ultrasonic detector, 4,518 passes of 9 species and 4 genera were recorded. In the DFE area, we recorded 12 species and 4 genera, in the FPE area, 10 species and 4 genera. The analysis did not show significant differences for the activity between DPE and FPE areas, however seasonal differences were found. The 19:00 and 20:00 hrs in both conditions registered the greatest number of passes. It is possible that environmental and landscape variables, which have been reported of importance for bat activity, intervene in this region as no differences were found in the analysis performed.

(C) 2017 Universidad Nacional Autónoma de México, Instituto de Biología. This is an open access article under the CC BY-NC-ND license (http://creativecommons.org/licenses/by-nc-nd/4.0/).

Keywords: Anabat; Ultrasonic detection; Feeding buzz; Activity times; Tehuantepec isthmus

\footnotetext{
* Autor para correspondencia.

Correo electrónico: margarita@ garcialuis.net (M. García-Luis).

La revisión por pares es responsabilidad de la Universidad Nacional Autónoma de México.
} 


\section{Introducción}

En los últimos años el desarrollo urbano se ha incrementado rápidamente, lo que provoca una creciente demanda de energía. En la actualidad, esta es generada principalmente a través de combustibles fósiles, lo que conlleva la emisión de gases de efecto invernadero, causantes primordiales del calentamiento global (McKinney, 2002). Una de las alternativas es la obtención de energía renovable, como la eólica, la cual ha tenido auge en los últimos años (Atienza, Martín-Fierro, Infante, Valls y Domínguez, 2011; Henestroza, 2008; Moryarty y Honnery, 2012). No obstante, se han registrado algunas consecuencias negativas, principalmente en ambientes templados, por el establecimiento y funcionamiento de parques eólicos, entre las que destacan la muerte de aves y murciélagos por colisiones, los ruidos (audible y ultrasónico) y el electromagnetismo (Cryan y Barclay, 2009; Henestroza, 2008; Kunz et al., 2007a).

Los estudios del impacto de los aerogeneradores sobre la fauna se han enfocado principalmente a las aves y recientemente a investigar las afectaciones a los murciélagos (Arnett et al., 2008). La metodología a nivel mundial se enfoca al conteo de cadáveres por aerogenerador por año o por megawatt generado (Hayes, 2013). La actividad de los murciélagos se ha evaluado a través de cámaras infrarrojas y detectores ultrasónicos; con estas técnicas se han determinado las diferencias de actividad por temporadas y su relación con la cantidad de cadáveres encontrados (Baerwald y Barclay, 2009; Horn, Arnett y Kunz, 2008; Johnson, Matthew, Wallace y Dale, 2004). Las investigaciones registran que las poblaciones de vespertiliónidos y molósidos que vuelan a grandes alturas tienen mayores impactos (Barros, Gastal y Rui, 2015; Kunz et al., 2007b).

En regiones tropicales de Latinoamérica, sobre todo en México y Brasil, las evaluaciones en los impactos sobre murciélagos son escasas, a pesar del creciente desarrollo y aprobación de proyectos de parques eólicos (Barros et al., 2015; Bolívar-Cimé, Bolívar-Cimé, Cabrera-Cruz, Muñoz-Jiménez y Villegas-Patraca, 2016; Rodríguez, Tiscornia y Olivera, 2009; Rodríguez-Durán y Feliciano-Robles, 2015; Zolotoff-Pallais, Cisneros, Mendieta y Medina, 2012). Debido a las diferencias en diversidad de la región neotropical con la región neártica (mayor riqueza y menor abundancia; Jenkins, Pimm y Joppa, 2013), son necesarios estudios poblacionales, de diversidad, riqueza y patrones de actividad espacial y temporal para evaluar de manera más precisa el efecto que tienen los parques eólicos sobre los murciélagos en ambientes tropicales.

En este estudio se analizan las diferencias en la riqueza de murciélagos artropodívoros (Segura-Trujillo, Lidicker y Álvarez-Castañeda, 2016) y sus patrones de actividad en sitios dentro y fuera de parques eólicos por temporada. Se ha reportado que los murciélagos son sensibles a la perturbación y responden generalmente con la disminución en riqueza y abundancia (Jung y Kalko, 2010; Stahlschmidt y Brül, 2012; Williams y Perfecto, 2011). Por lo tanto, predecimos que la riqueza y la abundancia de los murciélagos artropodívoros serán menores en las áreas con presencia de aerogeneradores, mientras que la actividad será mayor en sitios sin aerogeneradores y estará acotada a las primeras horas del atardecer, debido a que en ese periodo hay una mayor disponibilidad de presas (Rydell, Entwistle y Racey, 1996). La información generada podría ser utilizada para crear estrategias que disminuyan colisiones futuras de estos mamíferos en parques eólicos del neotrópico y apoyar el establecimiento de una mejor política de manejo y conservación.

\section{Materiales y métodos}

El área de estudio se ubica al sureste de México, en el istmo de Tehuantepec, Oaxaca. Es una de las zonas de mayor potencial eólico del país y del mundo, con vientos promedio de 7.7-8.5 m/s a $50 \mathrm{~m}$ sobre el suelo, calificados como excelentes para la producción de energía eólica y presentes durante todo el año (Elliott et al., 2004; fig. 1). El clima es cálido subhúmedo (Aw) con una temperatura media anual de $22-26^{\circ} \mathrm{C}$. La precipitación media anual va de 1,000 a 1,200 mm (Trejo, 2004). En esta región existen grandes extensiones de terrenos con alto grado de fragmentación, utilizados para agricultura de riego y temporal y cría de ganado bovino. Posee escasos corredores de vegetación arbórea con huizaches (Acacia sp.) y huamúchiles (Phithecellobium dulce), y con pequeños remanentes de bosque tropical caducifolio. El mayor afluente de la zona es el río Tehuantepec. Además, existen canales de riego para irrigar los pastizales y cultivos agrícolas, principalmente de maíz y sorgo (Inegi, 2013).

Dentro de la zona de estudio se seleccionaron 2 áreas con diferente condición. La primera dentro de parques eólicos (DPE, 20 parques en la región, con un total de 1,882 aerogeneradores; Sener, 2016) y la segunda fuera de parques eólicos (FPE, fig. 1).

\section{Diseño de muestreo}

Se establecieron 8 sitios de muestreo, 4 DPE (1 al 4) y 4 FPE (5 y 8). Desafortunadamente, durante el estudio 2 de los sitios FPE fueron invadidos por un nuevo parque eólico, razón por la cual solo se pudieron considerar datos de 2 sitios FPE (5 y 6). El trabajo se realizó en 2 ciclos de muestreo anuales durante la temporada seca (marzo a mayo) y la temporada lluviosa (junio a agosto) de 2013 y 2014, dando un total de 12 periodos de captura.

Para la recolecta de murciélagos con redes de niebla se utilizaron 8 y 12 redes $\left(12 \times 2.6 \mathrm{~m}\right.$; Avinet ${ }^{\circledR}$ Inc $)$ durante 2 noches por sitio por mes. Las redes se colocaron a partir del crepúsculo vespertino $(18: 00 \mathrm{~h})$ y permanecieron abiertas alrededor de $7 \mathrm{~h}$ diarias en posibles rutas de vuelo y en fragmentos de vegetación. El número total de noches para los sitios DPE fue de 48, mientras que para los sitios FDP fue de 24.

Los murciélagos capturados fueron identificados utilizando claves especializadas (Álvarez, Álvarez y López, 1994; Medellín, Arita y Sánchez, 2007). Se obtuvieron datos de edad (joven o adulto de acuerdo al grado de fusión de las falanges; Anthony, 1988), sexo y condición reproductiva (testículos escrotados, testículos inguinales, inactivo/preñada, lactante, poslactante); posterior a ello, los organismos fueron liberados en el mismo sitio de su captura. Algunos especímenes fueron recolectados y preparados como ejemplares de museo, principalmente molósidos y vespertiliónidos, de los cuales no se tuvo certeza de 


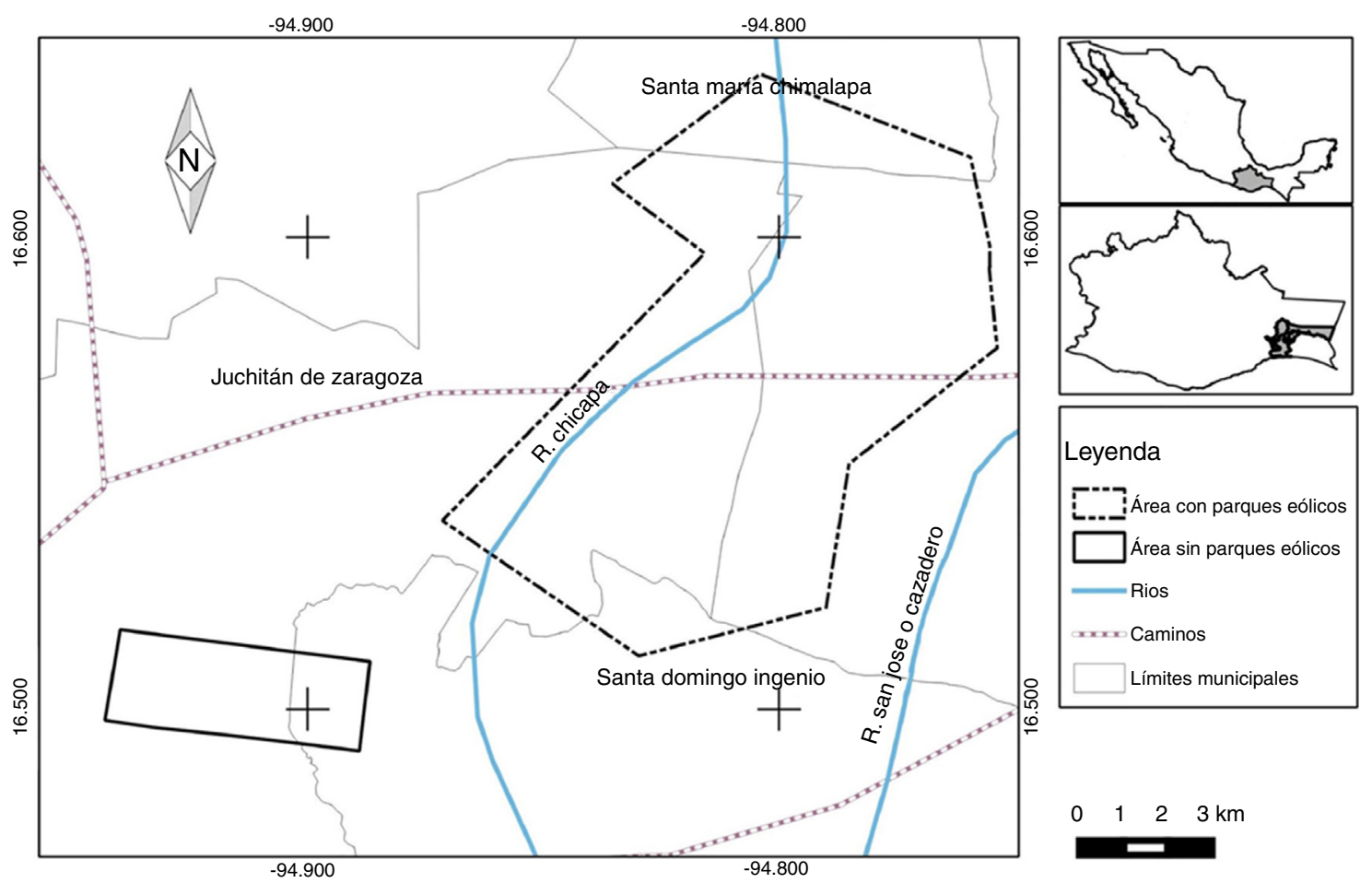

Figura 1. Localización de las áreas de estudio dentro de parques eólicos (DPE) y fuera de parques eólicos (FPE) en el istmo de Tehuantepec, Oaxaca, México.

identificación al 100\% en campo y de los que se obtuvieron grabaciones vocales. Los ejemplares de museo se depositaron en la Colección Mastozoológica (OAX.MA.026.0497) del Centro Interdisciplinario de Investigación para el Desarrollo Integral Regional, Unidad Oaxaca (CIIDIR-OAXACA, IPN). La captura fue realizada bajo el permiso de colecta (Semarnat, FAUT-0037), otorgado a los autores por las autoridades correspondientes (Secretaría de Medio Ambiente y Recursos Naturales).

La grabación de firmas vocales de murciélagos se llevó a cabo con el detector de ultrasonido Anabat SD2 (Titley Electronics, Ballina, Australia; intervalo de frecuencia 4-200 kHz, sistema de división de frecuencia). El detector funcionó aproximadamente $8 \mathrm{~h}$ al día, a partir del crepúsculo (18:00 h), y hasta la 01:59 am, con una noche de grabación por sitio por mes. En algunos sitios no se completaron las horas de grabación debido a condiciones ambientales, presencia de lluvia o vientos $>30 \mathrm{~m} / \mathrm{s}$. El método de grabación fue pasivo modificado del propuesto por O'Farrell, Miller y Gannon (1999) y Weller y Zabel (2002). El detector se colocó con una inclinación de $45^{\circ}$ a una altura de $30 \mathrm{~cm}$. Los archivos obtenidos con el Anabat SD2 fueron analizados con el programa AnalookW versión 3.8 (Titley Scientific, Ballina, Australia).

El análisis consistió en la separación de los archivos útiles. Se consideró como archivo útil (un pase) aquel que tuviera al menos 2 llamados sucesivos de ecolocalización; de esta forma se eliminaron archivos con secuencias fragmentadas o con ruido ambiental (Fenton, 1970; Thomas, 1988). Por otro lado, los eventos de caza (EC) fueron determinados a partir de los trenes de alimentación (definidos como un aumento en la tasa de repetición de llamados; Griffin, Webster y Michael, 1960), que indican la posible captura de presas en cada sitio. Se consideró cada tren de alimentación como un evento independiente (MacSwiney, Clarke y Racey, 2009). Finalmente, se determinaron los horarios y picos de actividad de las familias de murciélagos identificadas; los horarios de actividad se determinaron a través del número de pases por noche agrupados en intervalos de una hora.

La identificación de especies se efectuó, en el caso de las especies de la familia Mormoopidae, con grabaciones de referencia de obtención propia. Las grabaciones se obtuvieron de individuos capturados en refugios cercanos a la zona de estudio $(<10 \mathrm{~km}$ de distancia) a través del método de liberación en mano (hand release) en áreas abiertas (e.g., Frick, 2013; Williams y Perfecto, 2011). Las especies restantes fueron identificadas por comparación de espectrogramas de regiones o estados del país y de localidades de Centroamérica (Gannon, O'Farrell, Corben y Bedrick, 2004; Gannon, Sherwin, DeCarvalho y O'Farrell, 2001; Jung, Kalko y Von Helversen, 2007; O'Farrell, 1997; O'Farrel y Miller, 1997; Orozco-Lugo, GuillénServent, Valenzuela-Galván y Arita, 2013; Rydell, Arita, Santos y Granados, 2002). En algunos casos no fue posible llegar a nivel de especie, por lo que se analizaron a nivel de género, o también considerados como sonotipos (grupos de especies con llamados similares; Bader et al., 2015), particularmente para las familias Molossidae y Vespertilionidae, que poseen patrones de sonido variables o pulsos similares entre especies (Szewczak, 2000; Walters et al., 2012).

Para la determinación taxonómica de especies, o en su caso de los sonotipos, se midieron 6 parámetros de cada llamado (Dur: duración, Fmax: frecuencia máxima, Fmin: frecuencia mínima, fmean: frecuencia media, Tk: tiempo de inicio del llamado a la inflexión o rodilla, y Fk: frecuencia de la inclinación o rodilla; O'Farrell et al., 1999). La visualización de llamados se realizó 

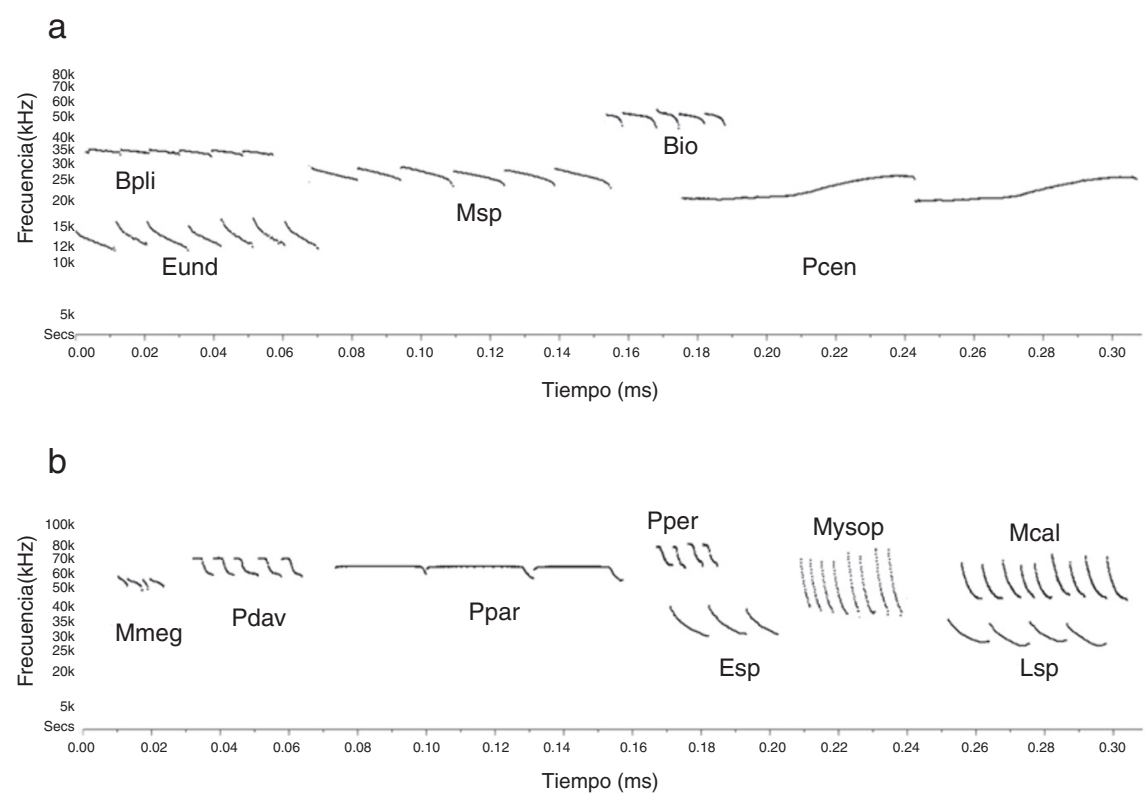

Figura 2. Espectrogramas de las especies registradas a través del detector ultrasónico Anabat SD2 en el área DPE y FPE en el istmo de Tehuantepec, Oaxaca, México. Visualización de espectrogramas, eje y en kiloHertz (kHz), eje x en tiempo (10 ms).

a) Familia Emballonuridae, Balantiopteryx io: Bio, Balantipteryx plicata: Bpli; familia Molossidae, Eumops underwoodi: Eund, Molossus sp: Msp, Promops centralis: Pcen.

b) familia Mormoopidae, Mormoops megalophylla: Mmeg, Pteronotus davyi: Pdav, Pteronotus parnellii: Ppar, Pteronotus personatus: Pper; familia Vespertilionidae, Eptesicus sp: Esp, Lasiurus sp.: Lsp, Myotis sp: Myosp, Myotis califonicus: Mcal.

en una división de frecuencia 16, con el eje de tiempo (x) en intervalos divididos cada $10 \mathrm{~ms}$ y eje de frecuencia (y) en escala logarítmica $(\mathrm{kHz})$.

\section{Análisis de datos}

Se generaron curvas de acumulación de especies y se determinó la eficiencia del muestreo para la captura en redes de niebla y detección ultrasónica. Por cada método de recolecta se realizaron matrices con los datos de presencia-ausencia, DPE y FPE, y se ingresaron al programa EstimateS ver.9.1 (Colwell, 2009). Los datos se combinaron 1,000 veces para obtener una distribución aleatoria, eliminar el efecto de orden de ingreso y suavizar la curva. La riqueza esperada se calculó a través de los estimadores no paramétricos Bootstrap y Chao 2. La completitud del inventario se obtuvo calculando el porcentaje de especies observadas respecto a las calculadas por los estimadores (Moreno y Halffter, 2000).

El esfuerzo de muestreo en redes de niebla se calculó por cada periodo para ambas temporadas (lluvias y secas) de 2013 y 2014. El total fue utilizado para estimar la abundancia relativa (AR), que se refiere al número de individuos colectados de cada especie, dividido por el número de m.red/hora total (Medellín, 1993). Para determinar diferencias en la captura de individuos con redes DPE y FPE y por temporadas se realizó la prueba no paramétrica de Kruskall-Wallis, por no cumplirse los supuestos de normalidad de los datos, a través del programa Infostat (Di Rienzo et al., 2016).

Para el análisis de los pases grabados y EC se realizó un modelo lineal generalizado (MLG; programa SPSS ver. 22) con la función de enlace Poisson y el estadístico de Wald (Mangeaud y Videla, 2005); la variable explicativa fue el número de conteos por cada sitio con 12 repeticiones (meses), es decir, 48 repeticiones para la condición DPE y 24 para FPE. Se compararon estos 2 factores (DPE, FPE) y se realizó la selección de modelos mediante el criterio de información de Akaike (AIC) y de máxima verosimilitud.

Finalmente, se usó una prueba de Kruskall-Wallis (Di Rienzo et al., 2016) para determinar diferencias entre los horarios de actividad con el programa Infostat. Para todos los casos se utilizó un nivel de significancia de $\mathrm{p}=0.05$.

\section{Resultados}

La riqueza general de especies de murciélagos artropodívoros de la zona estuvo compuesta por 13 especies y 4 géneros, agrupados en 4 familias. Se recolectaron 8 especies con redes de niebla, y se registraron 9 especies y 4 géneros por medio de detección ultrasónica (fig. 2, tablas 1 y 2). En el área DPE se registraron 12 especies y 4 géneros, mientras que en el área FPE resultaron 10 especies y 4 géneros. Pteronotus personatus y Eptesicus sp. se presentaron solo en DPE, mientras que Molossus rufus solo se registró en sitios FPE.

Para las redes de niebla, se obtuvo un esfuerzo de muestreo total de $127,872 \mathrm{~m} \mathrm{red} / \mathrm{h}$; para 2013 el esfuerzo fue de $55,296 \mathrm{~m}$ $\mathrm{red} / \mathrm{h}$, y $72,576 \mathrm{~m} \mathrm{red} / \mathrm{h}$ para 2014 . Por condición, en el área DPE el esfuerzo de muestreo fue de $85,240 \mathrm{~m} \mathrm{red} / \mathrm{h}$, mientras que para el área FPE fue de $42,624 \mathrm{~m} \mathrm{red} / \mathrm{h}$. En el caso de las temporadas, se registraron $63,936 \mathrm{~m}$ red/h para cada una.

Las curvas de acumulación de especies no alcanzaron la asíntota para ambas áreas. En el área DPE la riqueza estimada fue de 6.92 especies con Chao 2 y de 6.78 especies con Bootstrap 
Tabla 1

Registro de especies de murciélagos artropodívoros mediante detección acústica (A; número de archivos) y redes de niebla (RN; número de individuos), en sitios con presencia y ausencia de parques eólicos en el istmo de Tehuantepec, Oaxaca, México.

\begin{tabular}{|c|c|c|c|c|c|c|c|c|c|c|}
\hline \multirow[t]{2}{*}{ Especie } & \multirow[b]{2}{*}{ Método } & \multicolumn{4}{|c|}{ Dentro de parques eólicos (DPE) } & \multicolumn{4}{|c|}{ Fuera de parques eólicos (FPE) } & \multirow[b]{2}{*}{ Total } \\
\hline & & Ll-2013 & S-2013 & Ll-2014 & S-2014 & Ll-2013 & S-2013 & Ll-2014 & S-2014 & \\
\hline \multicolumn{11}{|l|}{ Familia Emballonuridae } \\
\hline Balantiopteryx io & A & 0 & $2(2)$ & 1 & 0 & 0 & 0 & 0 & 0 & $3(2)$ \\
\hline Balantipteryx plicata & A & 7 & 7 & 4 & 14 & 2 & 8 & 2 & 6 & 50 \\
\hline \multicolumn{11}{|l|}{ Familia Molossidae } \\
\hline Eumops underwoodi & A & 1 & 1 & 0 & 1 & 0 & 0 & 0 & 0 & 3 \\
\hline Molossus sp. & A & $193(3)$ & $151(88)$ & $218(2)$ & $102(1)$ & $52(4)$ & $142(1)$ & $215(4)$ & 146 & $1,219(103)$ \\
\hline Molossus molossus & $\mathrm{RN}$ & 0 & 1 & 1 & 2 & 0 & 2 & 0 & 1 & 7 \\
\hline Molossus rufus & $\mathrm{RN}$ & 0 & 0 & 0 & 0 & 0 & 0 & 0 & 1 & 1 \\
\hline Promops centralis & A & 2 & 0 & 0 & 3 & 0 & 0 & 0 & 2 & 7 \\
\hline \multicolumn{11}{|l|}{ Familia Mormoopidae } \\
\hline \multirow[t]{2}{*}{ Mormoops megalophylla } & A & 18 & 16 & 0 & 1 & 1 & 2 & 0 & 1 & 39 \\
\hline & RN & 2 & 2 & 0 & 2 & 0 & 45 & 0 & 0 & 51 \\
\hline \multirow[t]{2}{*}{ Pteronotus davyi } & A & $451(1)$ & $143(5)$ & 535 & 51 & 85 & 56 & $709(2)$ & 178 & $2,208(8)$ \\
\hline & $\mathrm{RN}$ & 1 & 0 & 0 & 0 & 0 & 9 & 2 & 0 & 12 \\
\hline \multirow[t]{2}{*}{ Pteronotus parnellii } & A & 16 & 4 & 35 & 7 & 1 & 44 & 1 & 10 & 118 \\
\hline & $\mathrm{RN}$ & 11 & 0 & 5 & 2 & 0 & 1 & 0 & 0 & 19 \\
\hline \multirow{2}{*}{ Pteronotus personatus } & A & 11 & 1 & 1 & 1 & 0 & 0 & 0 & 0 & 14 \\
\hline & $\mathrm{RN}$ & 0 & 0 & 0 & 0 & 0 & 1 & 0 & 0 & 1 \\
\hline \multicolumn{11}{|l|}{ Familia Vespertilionidae } \\
\hline Eptesicus sp. & A & 0 & $11(11)$ & 27 & 4 & 0 & 0 & 0 & 0 & $42(11)$ \\
\hline Lasiurus sp. & A & 21 & $31(11)$ & 15 & $34(1)$ & 15 & 1 & 12 & 5 & $134(12)$ \\
\hline Lasiurus intermedius & $\mathrm{RN}$ & 0 & 0 & 0 & 1 & 0 & 0 & 0 & 0 & 1 \\
\hline Myotis sp. & A & 61 & $116(17)$ & 72 & 42 & 32 & 20 & 152 & 122 & $617(17)$ \\
\hline Myotis califonicus & A & 3 & 0 & 0 & 0 & $60(1)$ & 0 & 1 & 0 & $64(1)$ \\
\hline Rhogeessa parvula & $\mathrm{RN}$ & 2 & 0 & 0 & 2 & 0 & 1 & 0 & 3 & 8 \\
\hline Individuos RN & $\mathrm{RN}$ & 16 & 3 & 6 & 9 & 0 & 59 & 2 & 5 & 100 \\
\hline Pases (EC) & A & $784(4)$ & 483 (134) & $908(2)$ & $260(2)$ & $248(5)$ & $273(1)$ & $1,092(6)$ & $470(0)$ & $4,518(154)$ \\
\hline
\end{tabular}

L1: temporada lluviosa; S: temporada seca.

Los números entre paréntesis representan los eventos de caza (EC).

El arreglo taxonómico siguió a Ramírez-Pulido, González-Ruiz, Gardner y Arroyo-Cabrales (2014).

(Sobs =6), por lo que se registraron el 86.71 y el $88.50 \%$ de la riqueza de especies estimada, respectivamente. Para el área FPE, con los estimadores Chao $2($ Sobs $=7$ y Sest $=8.38)$ y Bootstrap $($ Sobs $=7$ y Sest $=8.74)$ se registraron el 83.53 y el $80.09 \%$ de la riqueza de especies esperada (fig. 3b).

Se capturaron 100 individuos de 8 especies agrupados en 3 familias; la más capturada fue Mormoops megalophylla $(\mathrm{n}=51)$. Molossus rufus, P. personatus y Lasiurus intermedius se capturaron solo en una ocasión (tabla 2). En el área DPE se registraron 34 individuos, y Pteronotus parnellii y M. megalophylla tuvieron el mayor número de registros $(70.6 \%)$. En el área FPE se capturaron 66 individuos, M. megalophylla y Pteronotus davyi fueron las especies más capturadas (84.8\%). Al analizar las capturas entre las 2 temporadas, la mayor se observó durante la seca $(n=76)$ respecto a las lluvias $(n=24)$. En el análisis entre años, en el 2013 se registró el mayor número de ejemplares $(n=78)$ a diferencia de $2014(n=22$, tabla 1). Sin embargo, no se encontraron diferencias significativas por condición (DPE, FPE $)(\mathrm{H}=0.09$, g.l. $=1, p=0.75)$, ni por temporadas $(\mathrm{H}=3.89$, g.l. $=2, p=0.23)$.

Para la detección ultrasónica, las curvas de acumulación de especies para ambas áreas alcanzaron la asíntota en el doceavo mes de recolecta. La riqueza estimada en el área DPE fue de 13 especies con Chao 2 y de 13.54 con Bootstrap (Sobs = 13), por lo que se registró el $100 \%$ y $96.01 \%$ respectivamente de la riqueza de especies estimada. En el área FPE la riqueza estimada fue de 9 con Chao 2 y 9.42 con Bootstrap (Sobs =9) con registró de $100 \%$ y $95.54 \%$ respectivamente (fig. 3a).

Debido a que la prueba de Kruskall-Wallis no mostró diferencias significativas en el número de pases entre los sitios con aerogeneradores $(\mathrm{H}=4.37$, g.l. $=3, p=0.22)$ ni la prueba de Friedman entre los sitios sin ellos $\left(\mathrm{X}^{2}=1.60\right.$, g.l. $\left.=1, p=0.38\right)$, se trataron como 2 áreas independientes: dentro de parques eólicos (DPE) y fuera de parques eólicos (FPE).

Se obtuvieron 345 horas de grabación, 221 para el área DPE y 124 para el área FPE, en ambas se registraron 4,518 pases, que sirvieron para identificar 9 especies y 4 géneros pertenecientes a 4 familias (tabla 1). Para la familia Emballonuridae se identificaron 2 especies (Balantiopteryx io y Balantipteryx plicata). De la familia Molossidae se obtuvieron registros de 2 especies y 1 género (Eumops underwoodi, Promops centralis y Molossus sp.). Para la familia Mormoopidae se registraron 4 especies (Mormoops megalophylla, P. davyi, P. parnellii y P. personatus). En la familia Vespertilionidae se obtuvo una especie y 3 géneros (Myotis californicus, Eptesicus sp., Lasiurus sp. y Myotis sp.) (fig. 2a y b, tabla 2). 
Tabla 2

Abundancia relativa (AR, individuos.redes/h) de murciélagos capturados en redes de niebla y el promedio (X王 SD) de 6 parámetros medidos en los llamados de ecolocalización de las especies y géneros identificados a través del detector ultrasónico Anabat SD2 en el trópico mexicano.

\begin{tabular}{|c|c|c|c|c|c|c|c|}
\hline Especie & $\mathrm{AR}$ & Dur (ms) & Fmax $(\mathrm{kHz})$ & Fmin $(\mathrm{kHz})$ & Fmean $(\mathrm{kHz})$ & $\mathrm{Tk}(\mathrm{kHz})$ & $\mathrm{Fk}(\mathrm{ms})$ \\
\hline Balantiopteryx io & 0 & $6.42 \pm 1.87$ & $52.87 \pm 1.05$ & $45.68 \pm 1.26$ & $50.68 \pm 0.66$ & $0.41 \pm 0.39$ & $52.30 \pm 0.69$ \\
\hline Balantipteryx plicata & 0 & $7.24 \pm 1.52$ & $39.64 \pm 0.33$ & $38.41 \pm 0.51$ & $39.09 \pm 0.34$ & $0.24 \pm 0.22$ & $39.54 \pm 0.32$ \\
\hline \multicolumn{8}{|l|}{ Familia Molossidae } \\
\hline Eumops underwoodi & 0 & $9.72 \pm 1.70$ & $15.43 \pm 1.65$ & $11.94 \pm 0.50$ & $13.25 \pm 0.85$ & $2.51 \pm 1.28$ & $13.82 \pm 0.82$ \\
\hline Molossus sp. & 0 & $15.15 \pm 2.31$ & $27.34 \pm 0.80$ & $22.96 \pm 1.17$ & $25.36 \pm 0.89$ & $1.03 \pm 0.80$ & $26.95 \pm 0.93$ \\
\hline Promops centralis & 0 & $64.96 \pm 2.24$ & $25.95 \pm 0.34$ & $19.83 \pm 0.23$ & $22.43 \pm 0.09$ & $47.59 \pm 5.24$ & $24.56 \pm 0.84$ \\
\hline \multicolumn{8}{|l|}{ Familia Mormoopidae } \\
\hline Mormoops megalophylla & 0.000423 & $3.39 \pm 1$ & $55.86 \pm 1.24$ & $49.79 \pm 1.42$ & $53.23 \pm 1.8$ & $0.22 \pm 0.1$ & $55.18 \pm 0.81$ \\
\hline Pteronotus davyi & 0.000099 & $5.69 \pm 0.59$ & $72.26 \pm 0.49$ & $59.58 \pm 0.69$ & $66.46 \pm 0.74$ & $2.25 \pm 1.71$ & $66.69 \pm 4.75$ \\
\hline Pteronotus parnellii & 0.000157 & $27.47 \pm 3.01$ & $64.28 \pm 0.34$ & $59.11 \pm 1.70$ & $63.80 \pm 0.36$ & $2.11 \pm 0.86$ & $64.05 \pm 0.30$ \\
\hline Pteronotus personatus & 0.000008 & $2.01 \pm 1.37$ & $80.62 \pm 3.90$ & $72.69 \pm 7.61$ & $76.14 \pm 5.90$ & $0.51 \pm 0.63$ & $76.86 \pm 7.04$ \\
\hline Lasiurus intermedius & 0.000008 & 0 & 0 & 0 & 0 & 0 & 0 \\
\hline Myotis sp. & 0 & $3.58 \pm 0.81$ & $70.94 \pm 9.22$ & $41.65 \pm 2.17$ & $50.02 \pm 3.05$ & $2.25 \pm 0.57$ & $46.05 \pm 2.87$ \\
\hline Myotis califonicus & 0 & $5.24 \pm 0.83$ & $79.51 \pm 3.42$ & $52.80 \pm 0.79$ & $59.85 \pm 1.56$ & $2.83 \pm 0.57$ & $56.59 \pm 1.32$ \\
\hline Rhogeessa parvula & 0.000066 & 0 & 0 & 0 & 0 & 0 & 0 \\
\hline
\end{tabular}

Dur: duración; Fk: frecuencia de la inclinación o rodilla; Fmax: frecuencia máxima; Fmean: frecuencia media; Fmin: frecuencia mínima; Tk: tiempo de inicio del llamado a la inflexión o rodilla.

El arreglo taxonómico siguió a Ramírez-Pulido et al. (2014).
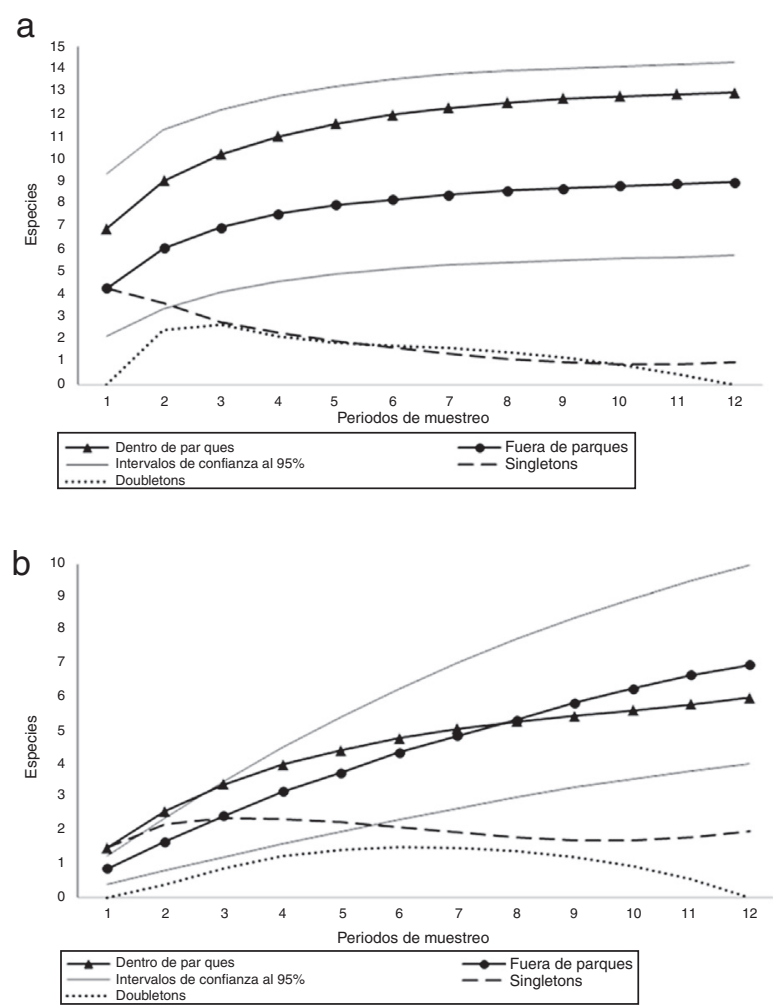

Figura 3. Curva de acumulación de especies por condición (DPE y FPE) en el istmo de Tehuantepec, Oaxaca, México.

a) Muestreo a través de detección ultrasónica.

b) Muestreo a través de redes de niebla.

Singletons: especie registradas en una ocasión; Doubletons: especie registradas en 2 ocasiones.
Al analizar los datos del número de pases, utilizando los factores DPE y FPE/temporada seca y temporada lluviosa, se generó solamente un modelo, por lo que no se reportan datos de Akaike (AIC) y devianza.

En el área DPE se identificaron 2,435 pases (54\%), que corresponden a 9 especies y 4 géneros. En el área FPE se obtuvieron 2,083 pases $(46 \%)$ de 6 especies y 3 géneros. No se observaron diferencias significativas de la actividad con el número de pases entre condiciones a través del MLG $\left(\chi^{2}\right.$ de Wald $=2.52, p=0.11$, tabla 3 ). Seis especies y 3 géneros son compartidos en ambas áreas, mientras que 3 especies y un género son exclusivos de los sitios DPE; el género que estuvo presente en ambas áreas a lo largo de todos los meses fue Molossus sp. (tabla 1).

Durante la temporada de lluvias 2013 se registró el $22.84 \%$ de pases y para secas, el 16.73\%. Para lluvias del 2014, el 44.27\% de pases, y para secas, el 16.16\%. Balantiopteryx plicata, Molossus sp., M. megalophylla, P. davyi, P. parnellii, P. personatus, Myotis sp. y Lasiurus sp. estuvieron presentes en ambas temporadas de 2013 y 2014 (tabla 1). Las diferencias en los porcentajes de pases registrados entre temporadas, tuvieron diferencias significativas de acuerdo al MLG ( $\chi^{2}$ de Wald $=4.50, \mathrm{p}=0.03$; tabla 3$)$.

En cuanto a los eventos de caza (EC), se registraron 154 pases para 3 especies y 4 géneros, los cuales estuvieron presentes en el 3.4\% de archivos del total. El género y la especie con más EC fue Molossus sp. $(\mathrm{n}=103,66.9 \%)$ y P.davyi $(\mathrm{n}=8,5.19 \%)$, respectivamente, en contraposición $M$. californicus y Eptesicus sp. con 1 y $11(0.65 \%$ y $7.14 \%)$ tuvieron el menor número de EC; mientras que 6 especies, no registraron EC (tabla 1). 
Tabla 3

Estimación de parámetros con un modelo lineal generalizado de distribución Poisson.

\begin{tabular}{|c|c|c|c|c|c|c|c|}
\hline \multirow[t]{2}{*}{ Parámetro } & \multirow[t]{2}{*}{ B } & \multirow[t]{2}{*}{ Error estándar } & \multicolumn{2}{|c|}{$95 \%$ de intervalo de confianza de Wald } & \multicolumn{3}{|c|}{ Contraste de hipótesis } \\
\hline & & & Inferior & Superior & $\chi^{2}$ de Wald & g.1. & Sig. \\
\hline (Interceptación) & 4.045 & 0.3017 & 3.453 & 4.636 & 179.771 & 1 & 0.000 \\
\hline [Condición B] & $0^{\mathrm{a}}$ & & & & & & \\
\hline [Temporada 1] & 0.713 & 0.3361 & 0.054 & 1.372 & 4.503 & 1 & 0.034 \\
\hline [Temporada 2] & $0^{\mathrm{a}}$ & & & & & & \\
\hline
\end{tabular}

Condición A: dentro de parques eólicos; Condición B: fuera de parques eólicos; Temporada 1: temporada de lluvias; Temporada 2: temporada de secas.

${ }^{a}$ Definido en cero porque este parámetro es redundante.

Tabla 4

Estimación de parámetros con un modelo lineal generalizado de distribución Poisson para eventos de caza (EC).

\begin{tabular}{|c|c|c|c|c|c|c|c|}
\hline Parámetro & B & Error estándar & \multicolumn{2}{|c|}{ 95\% de intervalo de confianza de Wald } & \multicolumn{3}{|c|}{ Contraste de hipótesis } \\
\hline (Interceptación) & 0.831 & 0.1707 & 0.497 & 1.166 & 23.725 & 1 & 0.000 \\
\hline [Condición B] & $0^{\mathrm{a}}$ & & & & & & \\
\hline [Temporada 1] & -1.183 & 0.1570 & -1.491 & -0.875 & 56.778 & 1 & 0.000 \\
\hline [Temporada 2] & $0^{\mathrm{a}}$ & & & & & & \\
\hline
\end{tabular}

Condición A: dentro de parques eólicos; Condición B: fuera de parques eólicos; Temporada 1: temporada de lluvias; Temporada 2: temporada de secas.

${ }^{a}$ Definido en cero porque este parámetro es redundante.

Se registró el mayor porcentaje de EC en el área DPE $(92.2 \%)$, en la temporada seca (88.96\%) y en el año 2013 (93.50\%). A partir del análisis con un MLG, se observaron diferencias significativas para condición $\left(\chi^{2}\right.$ de Wald $=28.49$, g.1. $\left.=1, \mathrm{p}=0.00\right)$ $\mathrm{y}$ temporadas $\left(\chi^{2}\right.$ de Wald $=56.77$, g.1. $=1, \mathrm{p}=0.00$; tabla 4$)$.

La representación de la actividad de los murciélagos por horas se agrupó por familia y área, dado que mostraba patrones similares por especies y por sitios (fig. 4). Para el área DPE se registró actividad a las 19:00 h y entre las 22:00 y las 23:00 h. En el área FPE se observaron 2 picos de actividad, a las 19:00 y entre a las 24:00 y la 01:00 h. La familia Emballonuridae tuvo escasa representación, por lo que no se observó un patrón definido. La Molossidae tuvo mayor actividad entre las 19:00 y las 20:00 h en ambas áreas. La Mormoopidae mostró mayor actividad en el área DPE de las 19:00 a las 20:00 h y de las 22:00 a las 23:00 h (fig. 4a). De manera similar, en el área FPE se registraron 2 picos de actividad (fig. 4b). La Vespertilionidae mostró actividad en el área DPE a lo largo de la noche, sin picos bien definidos; en contraste, en los sitios FPE se observó un pico a las 19:00 h. En el análisis de los horarios de actividad se observó que el área DPE, las 19:00 y las 20:00 $\mathrm{h}$ acumularon el 18.88 y el 11.04\% $(\mathrm{n}=853,499)$ de pases, respectivamente, mientras que en el área FPE las 19:00 h acumuló el $17.66 \%$ de pases $(\mathrm{n}=798)$. A través del análisis de los intervalos de actividad se encontró que hay diferencias significativas entre los pases registrados por hora $(\mathrm{H}=48.78$, g.l. $=7, \mathrm{p}=0.0001)$.

\section{Discusión}

La fauna de murciélagos en esta región está constituida por al menos 70 especies (Briones-Salas, Cortés-Marcial y Lavariega, 2015), de las cuales aproximadamente el $51 \%$ pertenecen al b
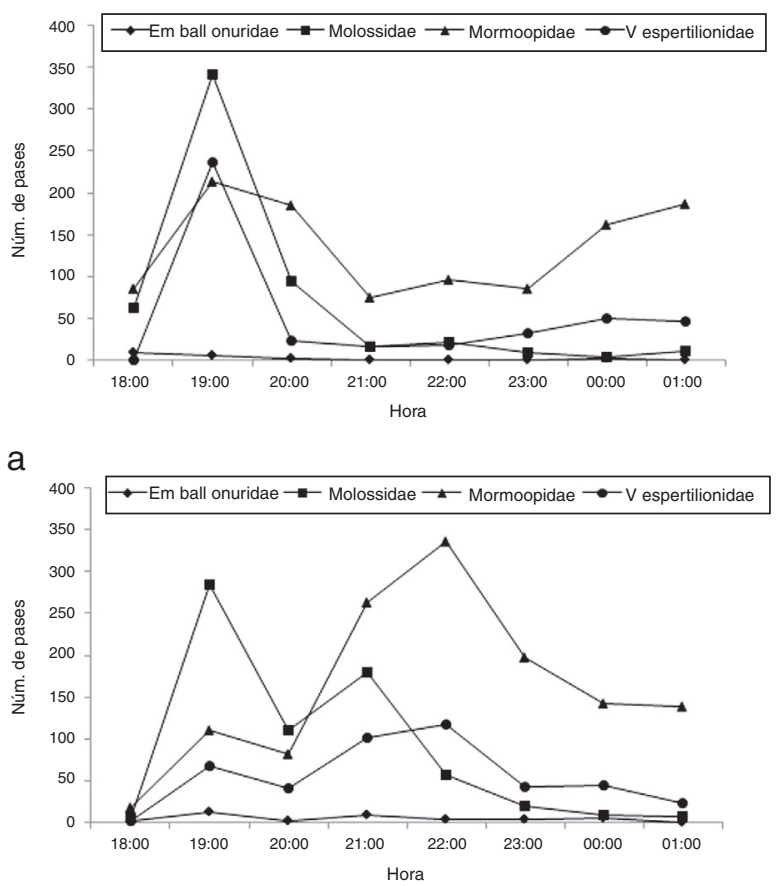

Figura 4. Horarios de actividad de las familias de murciélagos artropodívoros en el istmo de Tehuantepec, Oaxaca, México.

a) Sitios DPE. b) Sitios FPE.

gremio artropodívoro. Las especies y géneros registrados de este gremio en el estudio $(n=17)$ representan el $36 \%$ para la región. Bolívar-Cimé et al. (2016) registraron 20 especies de murciélagos artropodívoros en una zona con presencia de parques eólicos colindante a la zona de estudio; de estas, 10 coinciden con 
este estudio, 3 de nuestros registros no aparecen en sus listas y las especies restantes no se registraron. Esto posiblemente se deba a que el sitio de estudio de los autores antes mencionados se encuentra con una mayor cobertura vegetal, relativamente más cerca de un bosque tropical caducifolio en buen estado de conservación (Pérez-García, Meave y Salas, 2010).

En sitios con parques eólicos para Estados Unidos y Europa, el número de especies registradas mediante detección ultrasónica es menor que las reportadas aquí (Amorim, Rebelo y Rodrigues, 2012, 3 especies; Baerwald y Barclay, 2009, 5 especies; Fiedler, 2002, 6 especies; Minderman, Pendlebury, Pearce-Higgins y Park, 2012, 4 especies). Lo anterior es de esperarse dada la mayor riqueza de especies de la región neotropical, donde se ubica la zona con parques eólicos estudiada (Jenkins et al., 2013). No obstante, coincide con el número de registros obtenidos en los Chimalapas, Oaxaca, a $35 \mathrm{~km}$ al norte del área de estudio (9 especies y 4 géneros; Kraker-Castañeda, Santos-Moreno y García-García, 2013).

En estudios de la región neotropical, mediante el método de redes en Brasil, se identificaron 10 especies, 7 artropodívoras (Barros et al., 2015), mientras que en Puerto Rico se registraron 11, 5 artropodívoras (Rodríguez-Durán y Feliciano-Robles, 2015); en ambos estudios el número de especies identificadas es menor a las registradas en este trabajo. Lo anterior se atribuye al sesgo que tienen las redes en la captura de especies; se ha documentado que el uso de detectores de ultrasonido incrementa el registro de especies un 30 a 40\% (MacSwiney, Bolívar, Clarke y Racey, 2008; Pech-Canché, MacSwiney y Estrella, 2010). Esto resulta importante si se considera que la mayoría de las especies en las que se han registrado altas tasas de mortalidad por choque con las aspas de los aerogeneradores pertenecen a especies artropodívoras (Bolívar-Cimé et al., 2016; Kunz et al., 2007b). De la misma manera, se han realizado otros trabajos con mamíferos en la región del istmo de Tehuantepec, como el de Cervantes y Yépez (1995) (26 especies de murciélagos, 8 artropodívoras) y el de López, Lorenzo, Barragán y Bolaños (2009) (28 especies, 9 artropodívoras), que reportan más especies que las de este trabajo, debido a que no solo consideran registros resultado de trabajo de campo sino también registros de colecciones.

Destaca la presencia de $P$. davyi y el género Molossus como las especies más registradas en este trabajo. $P$. davyi se ha relacionado a vegetación de bosque (Jung y Kalko, 2011); sin embargo, el área de estudio posee escasa vegetación, y la presencia de esta especie puede atribuirse a la cercanía $(10 \mathrm{~km})$ con un área comunal protegida (Ojo de agua Cerro del Tolistoque), con remanentes de bosque tropical caducifolio en buen estado de conservación y que tiene una cueva que funciona como refugio de mormópidos y embalonúridos (Briones-Salas, Peralta-Pérez y García-Luis, 2013). Para el caso del género Molossus, esto coincide con lo encontrado por otros autores (Jung y Kalko, 2010, 2011; Kraker-Castañeda et al., 2013; Bolívar-Cimé et al., 2016), quienes encontraron que en sitios urbanos, pastizales y con parques eólicos en Gamboa (Panamá), Santa María Chimalapas y Juchitán (México) la actividad de molósidos es alta. Lo anterior se debe a que algunas especies de este género poseen cierta plasticidad adaptativa a condiciones de perturbación (Jung y Kalko, 2011). Además, es posible, que la presencia de parques eólicos permita que se mantengan en la zona, debido a que los miembros de esta familia habitualmente forrajean en áreas abiertas, necesarias para el óptimo funcionamiento de los aerogeneradores (Schnitzler y Kalko, 2001).

La mayor cantidad de registros de especies de las familias Mormoopidae y Molossidae en los sitios con parques eólicos coincide con lo reportado por otros autores en Brasil y Puerto Rico (Barros et al., 2015; Rodríguez-Durán y Feliciano-Robles, 2015) debido a la afinidad neotropical de las especies de esas familias; por otro lado, se contrapone con lo encontrado en parques eólicos de la región neártica, donde Vespertilionidae tiene mayor presencia (Amorim et al., 2012; Arnett et al., 2008).

La actividad de los murciélagos medida a través de pases no mostró diferencias significativas entre áreas, lo que se contrapone con lo esperado al inicio del trabajo. En otros estudios tampoco se han observado diferencias entre sitios con ciertos niveles de perturbación, como es el caso de lo observado (Kraker-Castañeda et al., 2013) en sitios con selva y pastizales y lo encontrado en áreas con fragmentos de bosque, bosque continuo y huertos con asentamientos humanos, en Veracruz, México (Estrada, Jiménez, Rivera y Fuentes, 2004).

El no encontrar diferencias significativas en la actividad entre condiciones podría responder a que ambas zonas de estudio (DPE y FPE) se ubican en una matriz de agricultura y ganadería con procesos significativos de fragmentación y pérdida en la cobertura vegetal. Estas condiciones se han mantenido desde hace más de 30 años, por lo que actualmente la vegetación nativa representa menos del 50\% en la zona (Muñoz, Villegas, MacSwiney y López-Acosta, 2016; Velázquez et al., 2003). Es posible que estas perturbaciones afectaran de manera importante la composición original de especies y solo sobrevivan aquellas que se adaptaron a estos cambios. Debido a lo anterior, la presencia de parques eólicos podría no tener grandes repercusiones en la actividad de los murciélagos, a diferencia de los cambios en la vegetación (e.g., Jung y Kalko, 2010, 2011). Adicionalmente, los resultados del análisis de actividad entre condiciones sugieren que el paisaje es usado de manera similar para el movimiento y la alimentación por las poblaciones de artropodívoros. Sin embargo, no se descarta que exista mortandad debido a choques con turbinas, y aunque en este estudio no se realizó búsqueda de cadáveres de murciélagos, en un parque eólico cercano al área de este estudio se registró una tasa de mortandad de 2.18-20.20 individuos por turbina y año (Bolívar-Cimé et al., 2016).

En el caso de las temporadas, la mayor actividad se presentó durante la temporada de lluvias, y es posible que esto se deba a que durante esta existe una mayor cantidad de alimento. Lo que se contrapone con lo encontrado en otros estudios (López-González, Lozano, Gómez-Ruiz y López-Wilchis, 2016; MacSwiney et al., 2009), que registraron una mayor actividad durante la temporada seca pero en sitios con presencia de cuerpos de agua, que puede atribuirse a la necesidad de hidratación de los murciélagos y a la disponibilidad de alimento.

Por otro lado, se obtuvo un alto porcentaje de EC $(n=154)$, comparado con lo obtenido en granjas del Reino Unido (1,747 pases, 47 EC; Wickramasinghe, Harris, Jones y Vaughan, 2003) y en la isla de Gotland en el mar Báltico (6 pases, 0 EC; Rydell y Wickman, 2015). En contraposición, se obtuvo un bajo porcen- 
taje de EC, si se compara con lo obtenido en sitios con cenotes y sin ellos en la región neotropical (2,111 pases, $665 \mathrm{EC}$ en total; MacSwiney et al., 2009). A pesar de que el área DPE registró casi la totalidad de EC y que la diferencia entre condiciones es significativa, el bajo número de estos en relación con otras regiones, sugiere que la zona es usada en mayor medida como un área de paso de las especies hacia otros sitios con mayores recursos, como canales y/o ríos. Esto porque los sitios de grabación no estuvieron cercanos o en cuerpos de agua, condición que ha sido reportada de importancia para los murciélagos, por la presencia de insectos de algunos de los cuales se alimentan (Fiedler, 2002).

Los horarios de actividad de los murciélagos en zonas con parques eólicos no han sido medidos anteriormente en el neotrópico. Los horarios de la familia Vespertilionidae y Molossidae estuvieron acotados a las primeras horas de la noche, lo que coincide con lo reportado por Agosta, Morton, Marsh y Kuhn (2005) en refugios en Maryland y Pennsylvania en EUA. En cuanto a la familia Mormoopidae, se observaron 2 picos de actividad, entre las 22:00 y las 23:00 h y entre las 12:00 y la 01:00 h. Es probable que un análisis detallado de la composición de la dieta de estas familias pudiera mostrar alguna relación con este comportamiento. Las diferencias de actividad entre horas coinciden con lo que se ha encontrado para la actividad de algunos insectos de los cuales se alimentan los murciélagos de estas familias (Rydell et al., 1996). Por tanto, al considerar que existe mortalidad DPE de acuerdo a lo encontrado en la región (Bolívar-Cimé et al., 2016) y que la actividad de murciélagos en el área de estudio se concentra en las primeras horas del atardecer y la noche (de 19:00 a 20:00 h y de 22:00 a 23:00 h), se sugiere la programación de altos al funcionamiento de los aerogeneradores en estos horarios, lo que podría beneficiar a las poblaciones de murciélagos estudiadas.

Finalmente, se considera que otros factores pueden influir sobre la actividad y los EC, por mencionar algunos: variables ambientales (viento, humedad y temperatura; Erickson y West, 2002; Lewis, 1993) y configuración del paisaje (presencia de caminos, cuerpos de agua, fragmentos de bosque, cultivos [Ávila-Flores y Fenton, 2005; Burles, Brigham, Ring y Reimchen, 2009]). Ello muestra futuras líneas de investigación en la zona, para descartar los posibles efectos de los aerogeneradores sobre las especies de murciélagos artropodívoros.

\section{Agradecimientos}

A las autoridades de los municipios de Juchitán de Zaragoza, Santo Domingo y Unión Hidalgo por permitirnos el acceso a sus terrenos. S. Peláez, Y. Santiago, M. Gómez, G. Medina, W. Juárez, O. Velasco, apoyaron el trabajo de campo y procesamiento de archivos. M. Lavariega, C. MacSwiney, R. Ávila-Flores y dos revisores anónimos realizaron comentarios y sugerencias sobre las primeras versiones del documento. M. Lavariega y C. Mases apoyaron en las pruebas estadísticas. Se agradece el apoyo de C. MacSwiney en la identificación de los llamados de Eumops underwoodi. El estudio fue apoyado parcialmente por la Secretaría de Investigación y Posgrado del IPN (SIP: 20140995) y por Acciona Energía México, S. de R.L. de C.V. MGL agradece al Conacyt por la beca núm. 351731 para la realización de sus estudios de doctorado y al IPN por la beca BEIFI. MBS fue apoyado por la Comisión de Operación y Fomento a las Actividades Académicas (COFAA), por el Programa de Estímulos al Desempeño Académico (EDI) y por el Sistema Nacional de Investigadores (SNI).

\section{Referencias}

Agosta, S. J., Morton, D., Marsh, B. D. y Kuhn, K. M. (2005). Nightly, seasonal, and yearly patterns of bat activity at night roosts in the Central Appalachians. Journal of Mammalogy, 86, 1210-1219.

Álvarez, T., Álvarez, C. S. T. y López, V. J. C. (1994). Claves para murciélagos mexicanos. México D.F.: Centro de Investigaciones Biológicas del Noreste, S.C./Escuela Nacional de Ciencias Biológicas, Instituto Politécnico Nacional.

Amorim, F., Rebelo, H. y Rodrigues, L. (2012). Factors influencing bat activity and mortality at a wind farm in the Mediterranean region. Acta Chiropterologica, 14, 439-457

Anthony, E. L. (1988). Age determination in bats. En T. H. Kunz (Ed.), Ecological and Behavioral Methods for the Study of Bats (pp. 47-58). Washington D.C.: Smithsonian Institution Press.

Arnett, E. B., Brown, W. K., Erickson, W. P., Fiedler, J. K., Hamilton, B. L., Henry, T. H., et al. (2008). Patterns of bat fatalities at wind energy facilities in North America. Journal of Wildlife Management, 72, 61-78.

Atienza, J.C., Martín-Fierro, I., Infante, O., Valls, J. y Domínguez, J. (2011). Directrices para la evaluación del impacto de los parques eólicos en aves y murciélagos (versión 3.0). Reporte. SEO/BirdLife, Madrid [consultado 25 Nov 2015]. Disponible en: https://www.aeeolica.org/uploads/documents/564-directricespara-la-evaluacion-del-impacto-de-los-parques-eolicos-en-aves-ymurcielagos_seo-birdlife.pdf.

Ávila-Flores, R. y Fenton, M. B. (2005). Use of spatial feature by foraging insectivorous bats in a large urban landscape. Journal of Mammalogy, 86, 1193-1204.

Bader, E., Jung, K., Kalko, E. K. V., Page, R. A., Rodriguez, R. y Sattler, T. (2015). Mobility explains the response of aerial insectivorous bats to anthropogenic habitat change in the Neotropics. Biological Conservation, 186, 97-106.

Baerwald, E. F. y Barclay, R. M. R. (2009). Geographic variation in activity and fatality of migratory bats at wind energy facilities. Journal of Mammalogy, 90, 1341-1349.

Barros, M. A. S., Gastal, M. R. y Rui, A. M. (2015). Species composition and mortality of bats at the Osório Wind Farm, Southern Brazil. Studies on Neotropical Fauna and Environment, 1, 1-9.

Bolívar-Cimé, B., Bolívar-Cimé, A., Cabrera-Cruz, S. A., Muñoz-Jiménez, Ó. y Villegas-Patraca, R. (2016). Bats in a tropical wind farm: Species composition and importance of the spatial attributes of vegetation cover on bat fatalities. Journal of Mammalogy, 97, 1197-1208.

Briones-Salas, M., Cortés-Marcial, M. y Lavariega, M. C. (2015). Diversidad y distribución geográfica de los mamíferos terrestres del estado de Oaxaca, México. Revista Mexicana de Biodiversidad, 86, 685-710.

Briones-Salas, M., Peralta-Pérez, M. y García-Luis, M. (2013). Acoustic characterization of new species of bats for the State of Oaxaca, Mexico. Therya, 4, 15-32.

Burles, D. W., Brigham, R. M., Ring, R. A. y Reimchen, T. E. (2009). Influence of weather on two insectivorous bats in a temperate Pacific Northwest rainforest. Canadian Journal of Zoology, 87, 132-138.

Cervantes, F. A. y Yépez, L. L. (1995). Species richness of mammals from the vicinity of Salina Cruz, coastal Oaxaca, México. Anales del Instituto de Biología, Universidad Nacional Autónoma de México, Serie Zoología, 66, 113-122.

Colwell, R. K. (2009). EstimateS ver. 8.2 [consultado 15 Nov 2015], Disponible en: http://viceroy. eeb.uconn.edu/EstimateS/Estimate SPages/AboutEstimateS.htm 
Cryan, P. M. y Barclay, R. M. R. (2009). Causes of bats fatalities at wind turbines: Hypotheses and predictions. Journal of Mammalogy, 90, 1330-1340.

Di Rienzo, J.A., Casanoves F., Balzarini, M.G., González, L., Tablada, M. y Robledo, C.W. (2016). Grupo InfoStat, FCA, Universidad Nacional de Córdoba, Argentina [consultado 21 Nov 2015]. Disponible en: http://www.cubasolar.cu/biblioteca/Energia/Energia52/HTML/Articulo06. htm

Elliott, D., Schwartz, M., Scott, G., Haymes, S., Heimiller, D. y George, R. (2004). Atlas de Recursos Éólicos del Estado de Oaxaca. Reporte, Laboratorio Nacional de Energía Renovable [consultado 8 Nov 2015],. Disponible en: https://www.nrel.gov/docs/fy04osti/35575.pdf.

Erickson, J. L. y West, S. D. (2002). The influence of regional climate and nightly weather conditions on activity patterns of insectivorous bats. Acta Chiropterologica, 4, 17-24.

Estrada, A., Jiménez, C., Rivera, A. y Fuentes, E. (2004). General bat activity measured with an ultrasound detector in a fragmented tropical landscape in Los Tuxtlas, Mexico. Animal Biodiversity and Conservation, 2, 5-13.

Fenton, M. B. (1970). A technique for monitoring bat activity with results obtained from different environments in southern Ontario. Canadian Journal of Zoology, 48, 847-851

Fiedler, J. K. (2002). Bat activity and mortality at Buffalo Mountain Windfarm eastern Tennessee (tesis doctoral). Knoxville, EUA: University of Tennessee.

Frick, W. F. (2013). Acoustic monitoring of bats, considerations of options for long-term monitoring. Therya, 4, 69-78.

Gannon, W. L., O'Farrell, M. J., Corben, C. y Bedrick, E. J. (2004). Call character lexicon and analysis of field recorded bat echolocation calls. En J. A. Thomas, C. F. Moss, y M. Vater (Eds.), Echolocation in Bats and Dolphins (pp. 478-484). Chicago: University of Chicago Press.

Gannon, W. L., Sherwin, R. E., DeCarvalho, T. N. y O'Farrell, M. J. (2001). Pinnae and echolocation call differences between Myotis californicus and $M$. ciliolabrum (Chiroptera Vespertilionidae). Acta Chiropterologica, 3, 77-91.

Griffin, D. R., Webster, F. A. y Michael, C. R. (1960). The echolocation of flying insects by bats. Animal Behaviour, 8, 141-154.

Hayes, M. (2013). Bats killed in large numbers at United States wind energy facilities. BioScience, 63, 975-979.

Henestroza, O. R. (2008). Desarrollo del proyecto eólico en la región del Istmo de Tehuantepec. Investigación y Ciencia de la Universidad de Aguascalientes, $42,18-21$.

Horn, J. W., Arnett, E. B. y Kunz, T. H. (2008). Behavioral responses of bats to operating wind turbines. Journal of Wildlife Management, 72, 123-132.

Inegi (Instituto Nacional de Estadística y Geografía) (2013). Mapas de uso del suelo y vegetación. Escala 1:250 000. Serie V. México [consultado 15 Ene 2016]. Disponible en: http://catalogo.datos. gob.mx/dataset/mapas-de-usodel-suelo-y-vegetacion-escala-1-250-000-serie-v-mexico

Jenkins, C. N., Pimm, S. L. y Joppa, L. N. (2013). Global patterns of terrestrial vertebrate diversity and conservation. Proceedings of the National Academy of Sciences of the United States of America, 110, E2602-E2610.

Johnson, G. D., Matthew, K. P., Wallace, P. E. y Dale, M. (2004). Bat activity, composition, and collision mortality at a large wind plant in Minnesota. Wildlife Society Bulletin, 32, 1278-1288.

Jung, K. y Kalko, E. K. V. (2010). Where forest meets urbanization: Foraging plasticity of aerial insectivorous bats in an anthropogenically altered environment. Journal of Mammalogy, 91, 144-153.

Jung, K. y Kalko, E. K. V. (2011). Adaptability and vulnerability of high flying Neotropical aerial insectivorous bats to urbanization. Diversity and Distributions, 17, 262-274.

Jung, K., Kalko, E. K. V. y von Helversen, O. (2007). Echolocation calls in Central American emballonurid bats signal design and call frequency alternation. Journal of Zoology, 272, 125-137.

Kraker-Castañeda, C., Santos-Moreno, A. y García-García, J. L. (2013). Riqueza de especies y actividad relativa de murciélagos insectívoros aéreos en una selva tropical y pastizales en Oaxaca, México. Mastozoología Neotropical, 20, 255-267.

Kunz, T. H., Arnett, E. B., Cooper, B. M., Erickson, W. P., Larkin, R. P., Mabee, T., et al. (2007). Assessing impacts of wind-energy development on nocturnally active birds and bats: A guidance document. Journal of Wildlife Management, 71, 2449-2486.
Kunz, T. H., Arnett, E. B., Erickson, W. P., Hoar, A. R., Johnson, G. D., Larkin, R. P., et al. (2007). Ecological impacts of wind energy development on bats: questions, research needs, and hypotheses. Frontiers in Ecology and the Environment, 5, 315-324

Lewis, S. E. (1993). Effect of climatic variation on reproduction by pallid bats (Antrozous pallidus). Canadian Journal of Zoology, 71, 1429-1433.

López, J. A., Lorenzo, C., Barragán, F. y Bolaños, J. (2009). Mamíferos terrestres de la zona lagunar del istmo de Tehuantepec, Oaxaca, México. Revista Mexicana de Biodiversidad, 80, 491-505.

López-González, C., Lozano, A., Gómez-Ruiz, E. P. y López-Wilchis, R. (2016). Activity of insectivorous bats is related to water availability in a highly modified Mexican temperate forest. Acta Chiropterologica, 18, 409-421.

MacSwiney, G. M. C., Bolívar, C. B., Clarke, F. M. y Racey, P. A. (2008). Insectivorous bat activity at cenotes in the Yucatán Peninsula, Mexico. Acta Chiropterologica, 11, 139-147.

MacSwiney, G. M. C., Clarke, F. M. y Racey, P. A. (2009). What you see is not what you get: The role of ultrasonic detectors in increasing inventory completeness in Neotropical bat assemblages. Journal of Applied Ecology, 45, 1364-1371

Mangeaud, A. y Videla, M. (2005). En busca de la independencia perdida: La utilización de modelos lineales generalizados mixtos en pruebas de preferencia. Ecología Austral, 15, 199-206.

McKinney, M. L. (2002). Urban, biodiversity, and conservation. BioScience, 52, 883-890.

Medellín, R. A. (1993). Estructura y diversidad de una comunidad de murciélagos en el trópico húmedo mexicano. En R. A. Medellín y G. Ceballos (Eds.), Avances en el estudio de los mamíferos de México (pp. 333-354). Ciudad de México: Asociación Mexicana de Mastozoología, A.C.

Medellín, R. A., Arita, H. T. y Sánchez, Ó. (2007). Identificación de los murciélagos de México clave de campo. México D.F.: Instituto de Ecología, UNAM.

Minderman, J., Pendlebury, C. J., Pearce-Higgins, J. W. y Park, K. J. (2012). Experimental evidence for the effect of small wind turbine proximity and operation on bird and bat activity. Plos One, 7, e41177.

Moreno, C. E. y Halffter, G. (2000). Assessing the completeness of bat biodiversity inventories using species accumulation curves. Journal of Applied Ecology, 37, 149-158.

Moryarty, P. y Honnery, D. (2012). What is the global potential for renewable energy? Renewable and Sustainable Energy Reviews, 16, 244-252.

Muñoz, J. O., Villegas, P. R., MacSwiney, G. M. C. y López-Acosta, J. C. (2016). Importancia de los elementos de un paisaje antropizado para la retención de diversidad de murciélagos en el istmo de Tehuantepec, Oaxaca, México. En A. Ramírez-Bautista y R. Pineda-López (Eds.), Fauna nativa en ambientes antropizados (pp. 115-122). Querétaro: Red Ambientes Antropizados (REFAMA).

O'Farrell, M. J. (1997). Use of echolocation calls for the identification of freeflying bats. Transactions of the Western Section of the Wildlife Society, 33, $1-8$.

O'Farrell, M. J. y Miller, B. W. (1997). A new examination of echolocation calls of some Neotropical bats (Emballonuridae and Mormoopidae). Journal of Mammalogy, 78, 954-963.

O'Farrell, M. J., Miller, B. W. y Gannon, W. L. (1999). Qualitative identification of free-flying bats using the anabat detector. Journal of Mammalogy, 80, $11-23$.

Orozco-Lugo, L., Guillén-Servent, A., Valenzuela-Galván, D. y Arita, H. T. (2013). Descripción de los pulsos de ecolocalización de once especies de murciélagos insectívoros aéreos de una selva baja caducifolia en Morelos, México. Therya, 4, 33-46.

Pech-Canché, J. M., MacSwiney, G. M. C. y Estrella, E. (2010). Importancia de los detectores ultrasónicos para mejorar los inventarios de murciélagos neotropicales. Therya, 1, 221-228.

Pérez-García, E., Meave, J. y Salas, S. (2010). Nizanda, Oaxaca. En G. Ceballos, L. Martínez, A. García, E. Espinoza, J. Bezaury, y R. Dirzo (Eds.), Diversidad, amenazas y áreas prioritarias para la conservación de las selvas secas del Pacífico de México (pp. 538-542). México, D.F.: Fondo de Cultura Económica-Comisión Nacional para el Conocimiento y Uso de la Biodiversidad (Conabio). 
Ramírez-Pulido, J., González-Ruiz, N., Gardner, A. L. y Arroyo-Cabrales, J. (2014). List of Recent Land Mammals of México. Lubbock, Texas: Museum of Texas Tech University.

Rodríguez, E., Tiscornia, G. y Olivera, L. (2009). Diagnóstico de las aves y mamíferos voladores que habitan en el entorno de la Sierra de los Caracoles y el diseño de un plan de monitoreo. Informe final. Maldonado, Uruguay: Administración Nacional de Usinas y Transmisiones Eléctricas (UTE) [consultado 8 Nov 2015]. Disponible en: http://portal.ute.com.uy/ sites/default/files/documents/files/institucional/GA\%20Informe $\% 20$ final $\%$ 20-\%20Diagnostico\%20y\%20Plan\%20de\%20Monitoreo.pdf

Rodríguez-Durán, A. y Feliciano-Robles, W. (2015). Impact of wind facilities on bats in the Neotropics. Acta Chiropterologica, 17, 365-370.

Rydell, J., Arita, H. T., Santos, M. y Granados, J. (2002). Acoustic identification of insectivorous bats (order Chiroptera) of Yucatan, Mexico. Journal of Zoology, 257, 27-36.

Rydell, J., Entwistle, A. y Racey, P. (1996). Timing of foraging flights of three species of bats in relation to insect activity and predation risk. Oikos, 76, 243-252.

Rydell, J. y Wickman, A. (2015). Bat activity at a small wind turbine in the Baltic Sea. Acta Chiropterologica, 17, 359-364.

Segura-Trujillo, C., Lidicker, W. Z., Jr. y Álvarez-Castañeda, S. T. (2016). New perspectives on trophic guils of arthropodivorous bats in North and Central America. Journal of Mammalogy, 97, 644-654.

Sener (Secretaría de Energía) (2016). Inventario Nacional de Energías Renovables (Inere). Secretaría de Energía [consultado 21 Mar 2016]. Disponible en: http://inere.energia.gob.mx.

Schnitzler, H. U. y Kalko, E. K. V. (2001). Echolocation by insect-eating bats. BioScience, 51, 557-569.

Stahlschmidt, P. y Brül, C. A. (2012). Bats as bioindicators - the need of a standardized method for acoustic bat activity surveys. Methods in Ecology and Evolution, 3, 503-508.
Szewczak, J. M. (2000). A tethered zip-line arrangement for reliably collecting bat echolocation reference calls. Bat Research News, 41 , 142.

Thomas, D. W. (1988). The distribution of bats in different ages of Douglas-fir forest. Journal of Wildlife Management, 52, 619-626.

Trejo, I. (2004). Clima. En A. J. García M, M. J. Ordoñez, y M. A. Briones (Eds.), Biodiversidad de Oaxaca (pp. 68-85). Oaxaca: Instituto de Biología, UNAM / Fondo Oaxaqueño para la Conservación de la Naturaleza / World Wildlife Fund.

Velázquez, A., Durán, E., Ramírez, I., Masa, J. F., Boccob, G., Ramírez, G., et al. (2003). Land use-cover change processes in highly biodiverse areas: The case of Oaxaca, Mexico. Global Environmental Change, 13 $175-184$.

Walters, C. L., Freeman, R., Collen, A., Dietz, C., Fenton, M. B., Jones, G., et al (2012). A continental-scale tool for acoustic identification of European bats Journal of Applied Ecology, 49, 1064-1074.

Weller, T. J. y Zabel, C. J. (2002). Variation in bat detections due to detector orientation in a forest. Wildlife Society Bulletin, 30, 922-930.

Wickramasinghe, L. P., Harris, S., Jones, G. y Vaughan, N. (2003). Bat activity and species richness on organic and conventional farms: Impact of agricultural intensification. Journal of Applied Ecology, 40, 984-993.

Williams, G. K. y Perfecto, I. (2011). Ensemble composition and activity levels of insectivorous bats in response to management intensification in coffee agroforestry systems. Plos One, 6, e16502.

Zolotoff-Pallais, J. M., Cisneros, C., Mendieta, R. y Medina, A. (2012). Diagnóstico del estado de composición de las poblaciones de aves (residentes-migratorias) y murciélagos en el complejo eólico «Eolonica», al sur de la ciudad de Rivas, Nicaragua. Temas Nicaragüenses, 86, 225-279. 